\title{
Prediction of Occupation Stress by Implementing Convolutional Neural Network Techniques
}

\author{
Surjeet Dalal, SRM University Delhi-NCR, Sonipat, India \\ Osamah Ibrahim Khalaf, Al-Nahrain University, Baghdad, Iraq
}

\begin{abstract}
Medicinal services experts experience significant levels of word-related worry because of their working conditions. Subsequently, the point of this study is to build up a model that spotlights human services experts in order to break down the impact that activity requests, control, social help, and acknowledgment have on the probability that a specialist will experience pressure. The authors have beforehand presented a technique for pitch highlight identification utilizing a convolutional neural network $(\mathrm{CNN})$ that yields great execution utilizing low-level acoustic descriptors alone, with no express span data. This paper utilizes this model for different pitch complement and lexical pressure discovery errands at the word and syllable level on the DIRNDL German radio news corpus. This research demonstrates that data on word or syllable span is encoded in the elevated level CNN include portrayal via preparing a direct relapse model on these highlights to foresee term.
\end{abstract}

\section{KEYWORDS}

Artificial Neural Network, Biomedical Signals, Deep Learning, Operational Stress

\section{INTRODUCTION}

Working pressure recognized as the pressure results from the recognition that the requests surpass one's ability to adapt at work. Wellsprings of working pressure consistently recognized since the 1970s. Components influencing worry at work incorporate physical condition, multifaceted nature of individual and gathering errands. Y. M. Ulrich-Lai (2009) depicted work stressors in association as occupation characteristics, staff connections, authoritative structure, physical characteristics, vocation improvement, and job changes in the association. Then again, S. Betti (2018) expressed that, classifications of stressors are assignment requests, physical requests and relational requests. Work assumes an amazing job in individuals' lives. It can either apply a significant impact on their prosperity which is an energizing background for some people, and can likewise be a gigantic wellspring of stress. Thusly, as work makes an ever increasing number of requests on schedule and vitality, individual progressively presented to both the positive and negative parts of business. The 
worker's connection among work and mental or physical wellbeing may add to profession alteration just as the efficiency and monetary practicality of the business.

Further R. Blaug (2007) discussed a detailed review of occupational stress related to physiological signal. An investigation led by a gathering of analysts proposed that, clashing situations are frequently ordinary occurrence which exists in both social and expert circles and spaces. As perceiving the contention is inescapable, there is an earnest need to handle this issue as needs be by perceiving and recognizing the underlying foundations of the issue at the beginning time. The trigger of pressure can be expected to psychosocial or physical requests of the workplace. A portion of the authoritative variables that may add to an expanded in anxiety are occupation related it is possible that they feel unbound with the position they are holding, tired of the long working hours or worry of move work. Some of them may have some relational clashes with associates or managers. Work place which is unsafe may make pressure certain individuals moreover. The amassed worry in an association if not settled at the beginning time may prompt low inspiration, non-appearance and medical issue which may prompt low profitability. Absence of authority over work, working environment, and business status recognized as wellsprings of stress and a basic wellbeing hazard for certain specialists. Representatives who can't apply command over their lives at work are bound to experience work pressure and in this way, bound to have weakened wellbeing. Numerous investigations secured that overwhelming position request and low control or diminished choice scope lead to work disappointment, mental strain, and cardiovascular malady. As a rule, work control is the capacity to apply impact over one's condition with the goal that the earth turns out to be all the more fulfilling and less undermining. People who have occupation control can impact the arranging and execution of work errands. Research found that it is the impact coming about because of support on certain obligatory exercises which influences occupation stress and wellbeing. For instance, G. E. Billman (2015) found that interest (participation at workforce conferences) negatively affected apparent employment stress, and a beneficial outcome on saw impact. This, thus, impacted enthusiastic strain, work fulfillment, non-appearance, and turnover expectation. So also, F. Lombardi (2011) reasoned that the capacity to control or impact work factors (e.g., speed and pacing of generation) connected to the frequency of cardiovascular illness just as to psychosomatic issue, work disappointment, and gloom. The accompanying rundown of impacts on one's workplace answered to significantly affect how adequately pressure tended to: support from collaborators the executives, acknowledgment accomplishment, high spirit, adaptable working conditions, and individual adapting techniques. These adapting systems incorporate; stress the executives' methods, adjusting proficient and private desires, unmistakably characterized jobs and setting up sensible gauges and desires. These discoveries give a convenient knowledge into the experience of worry inside colleges. Word related Stress Index (OSI) is a viable demonstrative apparatus for appraisal and consequent change of the workplace. The OSI is an added substance weight model which mirrors the viewpoint of intellectual ergonomics while fusing key parts of the main sociological work pressure models. Various scientists worldwide are at present utilizing OSI, and an enormous number of studies are on-going in which the OSI applied among expert drivers, doctors, instructors, and other word related gatherings. The OSI incorporated into the National Institute of Occupational Health and Safety (NIOSH) database of driving word related psychosocial appraisal apparatuses. This examination led to assess changing feelings of anxiety experienced by staff working in government colleges in Malaysia by utilizing OSI as an estimation apparatus. The finding of the examination will assist the businesses with redefining their procedure or helping college staff to survive or limit their feeling of anxiety. This examination proposed a usage procedure to address the present condition.

The impression of the individual are not considered in this methodology. As demonstrated in Figure 1, request put upon an individual (known as a stressor) surpasses 'as far as possible' of the individual's capacity to adapt or adjust to it.

Response-based model of pressure: The reaction based model rises up out of the field of prescription and is clarified from a physiological point of view. The physiological and mental 


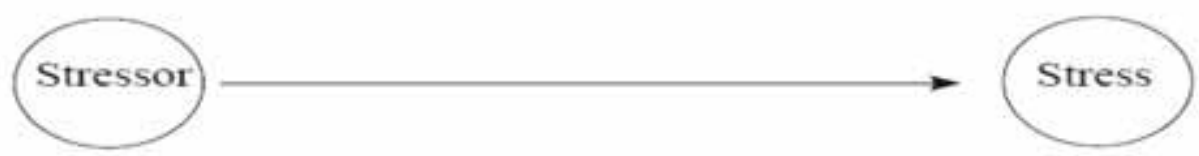

manifestations are not novel to push and can along these lines be credited to other ailments. Applied to the showing calling the teacher in this model is depicted as an inactive beneficiary who is pressurized by resultant pressure.

Figure 2 indicated the flow of response-based model of stress. It implies that various people, when gone up against with a similar circumstance, react in an unexpected way. The value-based methodology perspectives worry as inserted neither in the individual nor in nature, however in the interrelationship between the stressor, the person's view of the circumstance and their emotional reactions. In this definition the pretended without anyone else's input evaluation in deciding a person's feeling of anxiety is perceived. In an upsetting circumstance the individual assesses the circumstance (essential evaluation). Stress happens when the individual sees the circumstance as threatening to their significant objectives and feels incapable to satisfy these needs. Auxiliary examinations are made when the circumstance is made a decision as distressing. The individual decides the adapting assets in the auxiliary evaluation, which can prompt mental prosperity if no pressure is seen or sick wellbeing when stress is seen. Consequently, the experience of pressure emerges from teachers' view of requests, the powerlessness to satisfy those needs radiating from an absence of compelling adapting aptitudes and a definitive risk to the instructors' physical or mental prosperity. In this model instructors are on-screen characters and not latent beneficiaries of the outer weights.

This model recognizes, from one perspective, that instructing and a few schools specifically apply certain weights on the instructors and that teachers, then again, may respond in various approaches to carry an assortment of versatile assets to adapt to the stressors. From this model it very well may be seen that pressure is a mind boggling marvel. It is a procedure that includes various interfacing, value-based components enveloping stressors (natural requests), singular observations, the adapting assets accessible to the individual and the appearances of the requests. The reactions to the stressors are, nonetheless, affected by individual contrasts and methods for dealing with stress. It ought to likewise be noticed that there is a qualification between eustress (lovely pressure) and misery (horrendous pressure). Despite the fact that there might be likenesses between professionals' involvement of

\section{Figure 2. A response-based model of stress}

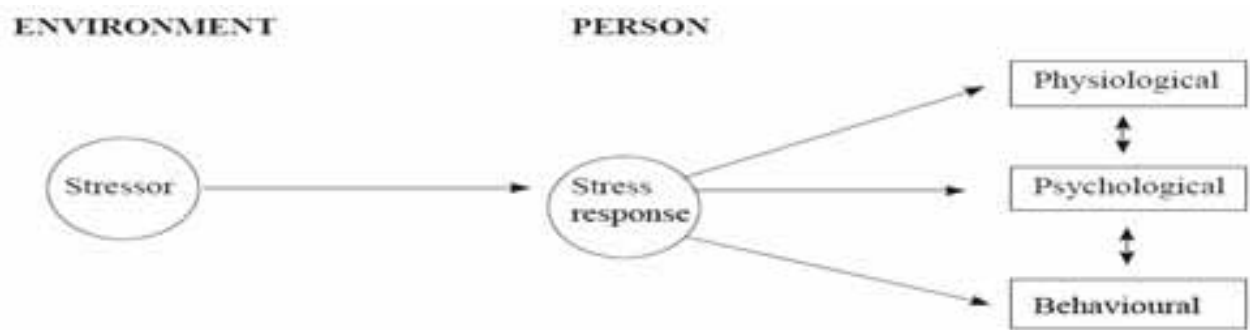


stress, it is significant with the end goal of this article to clarify word related pressure (trouble) as it happens in the instructing calling.

\section{LITERATURE REVIEW}

D. McDuff (2014) gauged physical wellbeing and passionate prosperity. Optional results incorporate skin maturing investigation, telomere shortening in anesthetists. 2 concentrated gatherings demonstrated tantamount statistic information and long stretches of work.

R. McCraty (2014) review that incorporated the dental staff of a therapeutic focus in Taiwan. They were approached to finish self-announced polls secretly. The Questionnaire on Medical Workers' Stress (QMWS) was utilized to evaluate work pressure, and the Maslach Burnout Inventory-Human Service Survey (MBI-HSS) was utilized to assess word related burnout. Altogether, 108 legitimate polls were gathered, with a reaction pace of 79.9\%. Information were broke down utilizing autonomous t-tests, single direction examination of change, Pearson's connection, and stepwise straight relapse. In general QMWS score demonstrated that meeting staff apparent altogether higher worry than postgraduate year occupants. Normal scores of MBI-HSS indicated dental associates' scores as like those of high burnout gatherings; visiting staff and moderate burnout gatherings had comparable scores. Stepwise relapse investigations uncovered that the noteworthy indicator of passionate depletion was the QMWS score, of depersonalization were filling in as a dental aide and QMWS score, and of individual achievement was holding an instructing position. Work pressure and word related burnout were basic among dental staff; this may influence tolerant security and ought to be esteemed. A pressure the board program is prescribed to advance emotional well-being of dental staff, alongside alteration of workplace, execution assessment, and advancement frameworks.

Y. R. Tabar (2017) decided the pervasiveness of a sleeping disorder, all out rest time and its sociostatistic corresponds in 799 medical caretakers working in mental and general emergency clinics in China, finding that the pervasiveness of at any rate one kind of detailed rest unsettling influence was $69.7 \%$; and the paces of trouble starting rest, trouble looking after rest, and early daytime arousing were $54.6 \%, 54.7 \%$, and $55.9 \%$, individually. The concentrate reasoned that the high feelings of anxiety looked by mental medical attendants contained the fundamental driver of their sleeping disorder.

N. Hjortskov (2007) stated that word related pressure is a discussion point for some, pros keen on enhancing the productivity of the workers working in various fields. In physical instruction and sports, this issue has a progression of explicit qualities dependent on the occupation (educator, mentor) necessities. This paper targets featuring the offer elements encouraging the event of stress contingent upon the occupation. Information investigation uncovered that word related pressure has explicit types of appearance. Results add to the showing calling monograph in the field and give reference focuses to individual and expert advancement projects intended to these classes of pros.

A. Moriguchi (1997) stated that word related pressure (OS) among instructors incline to gloom and tension. No investigation was done to survey these issues among Egyptian educators. This investigation intended to evaluate the pervasiveness of OS, gloom furthermore, uneasiness among Egyptian educators. A cross sectional examination was done on 568 Egyptian instructors.

The respondents filled a poll on close to home information, and the Arabic form of the Occupational Stress Index (OSI), the Arabic approved forms of Taylor show uneasiness scale and the Beck Discouragement Inventory (BDI) were utilized to survey OS, uneasiness and sorrow individually. The pervasiveness of OS, uneasiness and sorrow among instructors was $(100 \%, 67.5 \%$ and $23.2 \%$ ) individually. Operating system, uneasiness and sorrow scores were altogether higher among instructors with an age over 40 years, female instructors, grade teachers, those with insufficient compensation, higher showing background, higher capabilities also, higher outstanding task at hand. A critical powerless positive relationship was found between OS scores and uneasiness and misery scores. This investigation showed the requirement for future looks into to address hazard factors of 
OS and mental issue among Egyptian educators, and the need of periodical medicinal assessment of educators and medicinal and mental help for the recognized cases.

M. A. Oskoei (2008) presents the consequences of an investigation creating master framework to help pressure acknowledgment dependent on Artifical Neural Network. Sharma and Gedeon et al. (2010) researched characterization of worry in perusing for guys and females dependent on a counterfeit neural system model (ANN). A trial was directed with distressing and non-unpleasant perusing material as upgrades.

Q. Zhang (2018) structured a cell phone based framework, named mStress, utilizing 6 additional remote sensors to gather physiological information to help continuous discovery of stress. M. Brennan (2001) proposed Stress Sense for unpretentiously perceiving worry from cell phone recorded conversational voice information. Be that as it may, such applications depend on extra sensors or gadgets to gather one's genuine information. It makes stress recognition obtrusive to typical life, and can't be utilized broadly in more individuals. There are additionally a few specialists going to nonintrusive approaches to naturally identify mental worry from informal organizations. It displayed strategies to recognize mental worry from discussion posts and microblog tweets separately. These strategies are for the most part dependent on content information in the interpersonal organizations, while other similarly significant substance, like pictures and social activities are disregarded.

\section{OCCUPATIONAL STRESS INDEX (OSI)}

The OSI gets more from intellectual ergonomics and mind examine, endeavoring to portray, in quantitative terms, the weight of work forms upon the individual. The hidden inspiration for growing such a methodology is to help pinpoint zones for intercession, by endeavoring to reflect genuine work encounters.

There have been two noteworthy methodologies in word related psychosocial research utilizing self-report techniques. One has been to create occupation-explicit inquiries. This can give rich, nitty gritty data helpful in distinguishing key regions for intercession. The OSI speaks to a potential methods for connecting these two different methodologies. As expressed via Landsbergis and Theorell (Ibid): " An ongoing imaginative methodology utilized occupation-explicit inquiries (valuable for work environment intercessions), that depend on general inquiries. The Occupational Stress Index (OSI) can be custom fitted to explicit occupations, in this manner permitting examination among occupations of the pressure weight looked by laborers". We can make correlations with respect to the complete weight, just as in the idea of the word related pressure trouble. These inquiries are of intrigue in the examination setting, but at the same time are those explained by working individuals themselves.

\subsection{Deep Learning Network (DNN)}

Deep learning is a part of AI that began from counterfeit neural system has demonstrated extensive accomplishment in assorted fields in drug, business, government segments and so forth. It endeavors to display information progressively and orders examples utilizing different non-straight handling layers. Since late written works have shown the achievement of CNN profound learning models in the use of biomedical sign investigation, the focal point of this paper is constrained to inspecting the past writings identified with CNN models.

\subsection{Convolutional Neural Network (CNN)}

The best sort of profound learning model is convolutional neural systems (CNNs). CNNs were first structured by Fukushima in 1980 (Desai et al., 2016). Notwithstanding, the brilliant time of CNN began in the most recent decade. The principal development of CNNs was when AlexNet won the ImageNet rivalry. From that point forward, just systems use convolutional neural systems have won this challenge. The CNN is a kind of profound learning system which contains at least one convolutional and max pooling layers pursued by at least one completely associated layers, which is alluded to as 
the characterization layer. A case of a CNN is appeared in Figure 3. The CNN is unique in relation to the straightforward multi-layer arrange (MLP). MLPs just use information and yield layers, and, probably, a solitary shrouded layer, where in the profound inclining system there are multiple layers including information and yield layers.

Figure 3 indicated separates between a basic MLP and a CNN. Each square in the CNN contains various layers. The second key contrast between the CNN and MLP is the mix of pooling layers in the previous, where pixel esteems are collected utilizing a change work. This will diminish the quantity of parameters in the system. At long last, completely associated layers will be toward the part of the arrangement. In the completely associated layer, loads are never again imparted to the convolutional layer.

The difference between MLP and a CNN is highlighted by Figure 3. The principle points of interest of CNNs are that they are self-scholarly and self-sorted out systems without the requirement for supervision. Besides, the assignment of pre-preparing and highlight extraction strategies are not required in CNNs. Conversely, the profound learning system can naturally perceive progressively complex highlights in view of the quantity of convolutional layers it contains. This capacity of profound learning underpins the capacity of the system to deal with huge, high-dimensional information which contains countless highlights. This makes the CNN helpful and diminishes obligation during preparing and choosing of the best includes that separate classes in the dataset. As of now, a huge use of the CNN is in picture order, article or penmanship acknowledgment and discourse acknowledgment. What's more, it assumes a significant job in the biomedical field for robotized ailment finding. Likewise, Deep learning systems are additionally effectively used to applications requiring the preparing of enormous measures of information. Numerous applications that utilization the $\mathrm{CNN}$ have accomplished more noteworthy proficiency and execution for constant grouping.

\section{METHODOLOGY}

\subsection{Subjects}

In this research paper, out of 20 students, there exists of 8 females and 20 male in this group which ageing from 18 to 35 .

\section{Figure 3. The differences architecture between a simple MLP and a CNN}

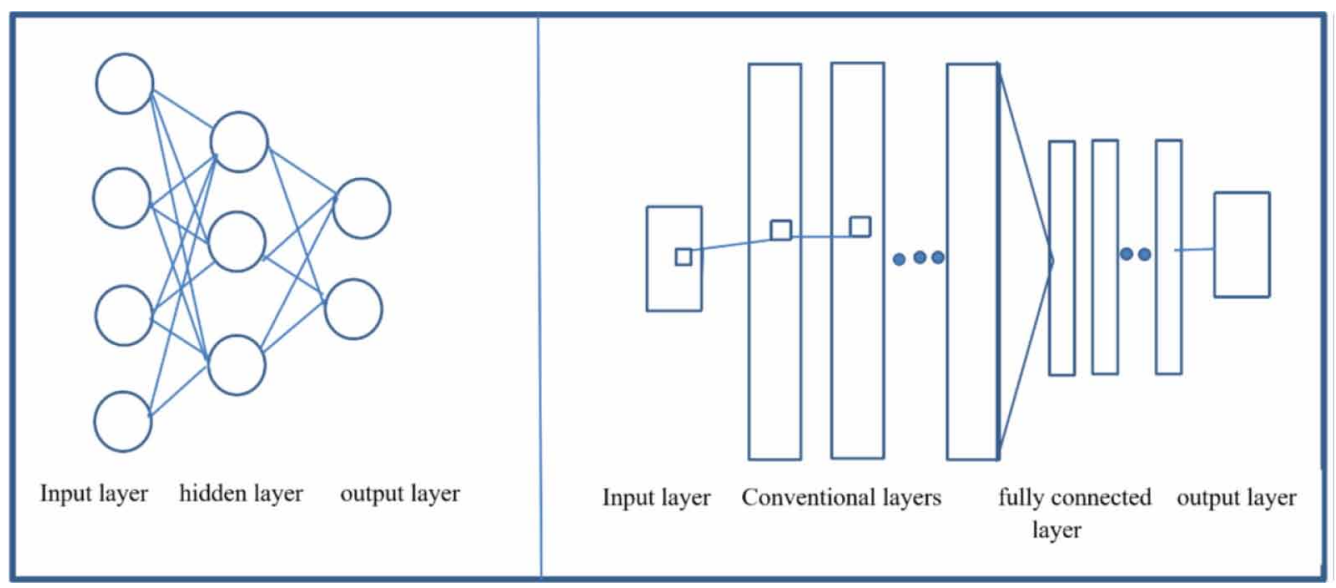


The group members are being selected on the basis of following factors as given below:

1. No past of nervous and coronary illness;

2. No sensitivity to cement or scouring liquor;

3. Getting optional school instruction or sophisticated, fit for accomplishment two-digit number juggling count rationally. Composed educated assent was gotten before the investigation.

Figure 4 demonstrate the experimental setup of the proposed work.

\subsection{Biomedical Signals}

Biomedical experts and researchers have concentrated on dissecting the presence of various kinds of time arrangement in the biomedical region for determination and identification purposes. For instance, biomedical researchers attempt to recognize maladies identified with mind works by observing natural sign from patients recording an electroencephalogram (EEG). The EEG sign give helpful data about the electrical movement of the mind. Nervous system specialists can analyze diverse unusual movement in the cerebrum by direct visual review. For instance, an EEG is a typically utilized in epilepsy conclusion.

The EEG records the sign from countless information channels. It very well may be from 12 to 256 anodes. Every one of these channels has a high worldly goals. Investigating this number of sign requires the improvement of online calculations that can manage the changing kinds of information. Another sort of biomedical sign is the electrocardiogram (ECG). Specialists have endeavored to record electrical exercises of the heart so as to distinguish coronary illness by utilizing an ECG. The sign are recorded by setting a terminal in the chest. From these sign, the unusual movement of the heart can

\section{Figure 4. Experimental Setup}

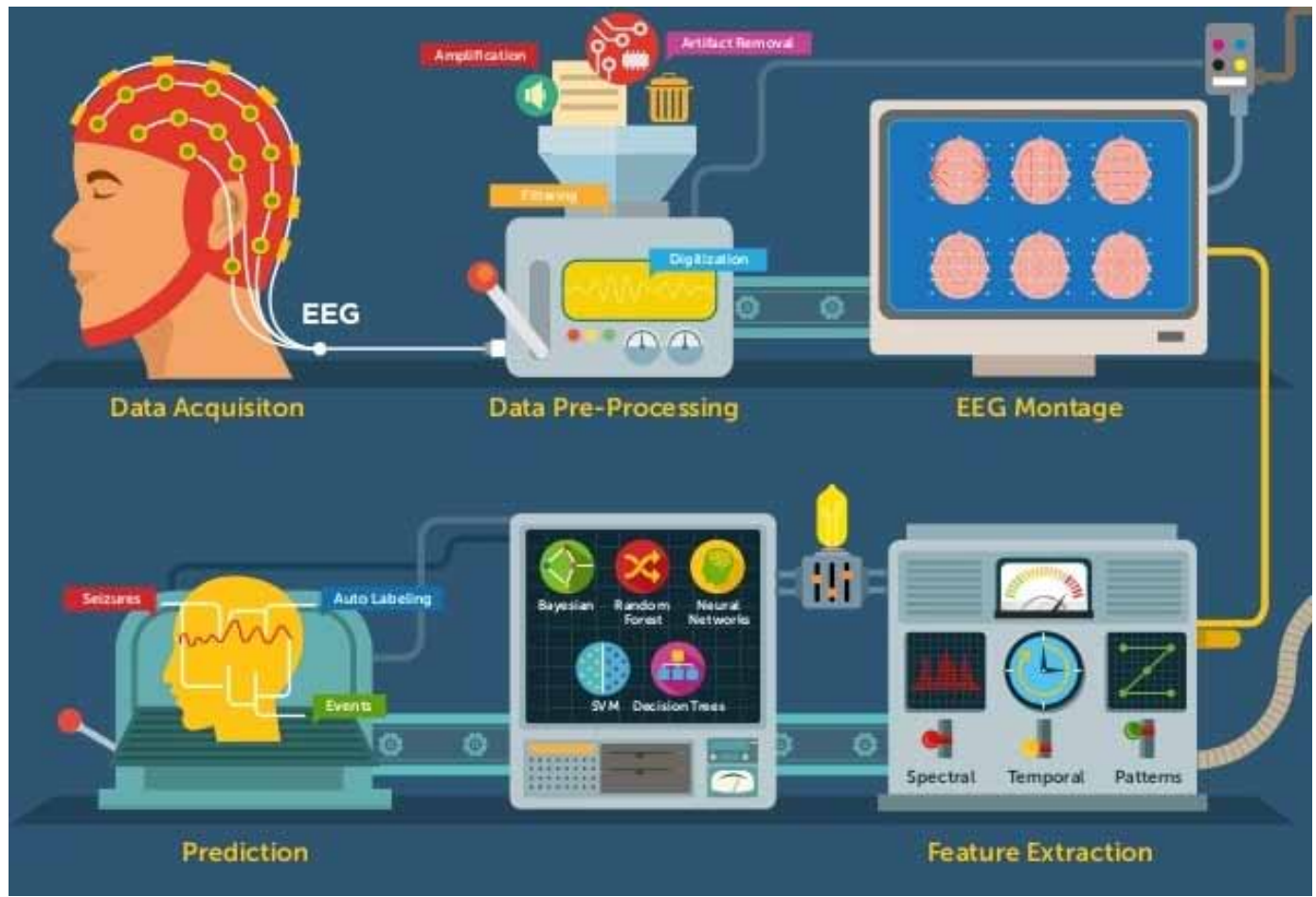


be identified. The ECG is a useful analytic device to distinguish different cardiovascular maladies. Electromyogram (EMG) is the other sort of biomedical sign. The EMG has been utilized to record the electrical action of muscle constrictions, and these sign have been recorded from various pieces of the human body so as to comprehend the body's practices under ordinary and obsessive conditions.

\subsection{Application of CNN for EEG Analysis}

The greater part of examines concentrated on naturally concentrate highlights from crude biomedical flag and accomplishes preferred arrangement over a component based methodology. The main examination that utilized a CNN for investigation of EEG sign was led by For instance crossrelationship, nonlinear association, contrast of Lyapunov examples and wavelet examination based synchrony, for example, stage locking are utilized to extricated highlights from EEG signals. These highlights were utilized to prepare three classifiers, for example, regularized strategic relapse, CNN, and SVM. The dataset utilized on this exploration on the standard Freiburg EEG dataset which recorded sign from 21 patients having medicinally recalcitrant central epilepsy. The CNN was accomplished $100 \%$ of the seizures all things considered an hour prior to the beginning. What's more, the CNN utilized REM Behavior Disorder (RBD) conclusion from the EEG. The EEG was recorded from RBD patients and sound controls. In their work, they chose a couple of minutes of eyes-shut resting state from EEG signals. The contribution to the CNN was spectrograms of EEG channel information, i.e., 2D time-recurrence maps. They contemplated a profound repetitive neural system utilizing stacked long momentary memory organize (LSTM) cells or gated-intermittent unit (GRU) cells. The proposed methodology accomplished $80 \%$ exactness. Most momentum research has concentrated on the acknowledgment of irregularity of EEG signals. Then again, it endeavored to apply a profound learning system to EEG sign to demonstrate clinical cerebrum demise conclusion. The Short Time Fourier Transform strategy was utilized as a period recurrence investigation procedure. At that point, spectrogram pictures were made to describe the highlights of EEG signals. For the analysis, EEG sign utilized in this paper were acquired from cerebrum harmed patients. These pictures were utilized as the preparation tests in the CNN. At long last, the prepared system was applied to evaluate the level of comparability of the other patients' EEG signals with those of the patients in a trance like state and with cerebrum demise manifestations. The outcome was $99.8 \%$ exactness. This methodology can be utilized to assess the state of cerebrum harmed patients just as for semi mind demise analysis (. Another examination concentrated on to recognize EEG example of three classes in particular ordinary, preictal and seizure design. In this examination, 13-convolutional layers were utilized to assemble the CNN so as to distinguish ordinary, preictal and seizure design. They accomplished a precision of $88.67 \%$, particularity of $90 \%$, and affectability of $95.00 \%$. Also CNN has been utilized to segregate distinctive sort of cadence types and furthermore to recognize the rhythms themselves. In this section, EEG accounts of musicality observation. The examination utilized EEG information recorded inside a beat recognition study in Kigali, Rwanda which contains 12 East African and 12 Western cadenced. $\mathrm{CNN}$ had the option to distinguish singular rhythms from the EEG with $24 \%$ precision.

\subsection{Application of CNN for EMG Analysis}

The ascent in the utilization of wearable gadgets as of late has expanded the capacity to catch a scope of different physiological and useful information. This information would now be able to be recorded ceaselessly for applications in games, welfare and social insurance. This immense measure of data needs powerful devices and investigation methods. Thusly, profound learning systems lead the pack to manage this high-dimensional information. A profound learning system was created to distinguish movement from inertial sensor information. They consolidated two arrangements of highlights. This blend defeated a portion of the restrictions in a regular profound learning engineering. They utilized lab and true movement datasets. Otherworldly area was used before the information is passed onto the profound learning system. The outcomes showed the capacity of the proposed way to deal with identify diverse human action, beating different strategies with exactness and the review is above $85 \%$. 
Besides, the investigation demonstrated that the calculation times for the proposed methodology are enduring with the confinements of constant preparing on cell phones and a wearable sensor. Then again, profound learning has been utilized for EMG hand development acknowledgment. They utilized a CNN model to discover six hand developments through EMG signals. The outcomes demonstrate a decent arrangement exactness for CNN contrasted with SVM. The best precision was arrived at utilizing CNN with $90 \%$ exactness. Also, a CNN was used for characteristic control of automated hands by EMG signals. The CNN was tried to group a normal of 50 hand developments in 67 flawless subjects and 11 transradial amputees. With this model the announced precision was $66.59 \%$. Additionally, there is study concentrating on order of telephones and phonetic highlights by utilizing facial EMG signals. The investigation concentrated on EMG-based quiet discourse recognizer. They expected to extricate EMG exercises for specific telephones, present and missing phonetic highlights. They accomplished a decent precision in one of their trials which was about $70 \%$ to identify Rounding word. In this way, a multi-stream $\mathrm{CNN}$ is applied to show that a little arrangement of muscles can impact on explicit hand developments. The CNN was racing to get familiar with the connection between's individual muscles and explicit signals. In this examination, multi-stream disintegration organize and the combination stage was utilized. Aftereffect of the model was recorded (from $77.8 \%$ to $85 \%$ ) precision.

\subsection{Feature Extraction}

Diagram of separated highlights and their little portrayal are given. Highlight separated for ECG and EMG sign were as per the procedure depicted in upcoming section. Highlights from EEG sign were separated. All sign handling were finished by MATLAB and BIOPAC AcqKnowledge programming. Normalizing of gathered highlights was utilized as a result of the huge fluctuation in gathered information of various subjects. Highlights were gathered over 120s sign at a uniform advance of first step level was not less in light of the fact that we expect that feeling of anxiety of the client was not evolving significantly.

\subsection{Classification Procedure}

So a complete 28 component were extricated from the gathered flag however it was normal that there will be critical relationship between the highlights as they were gathered dependent on same physiological procedure. A three layer Back Propagation Neural Network (NN) was organized for the better order of the gathered highlights and stress location.

Since here SS condition was isolated in to two sub classifications Stress because of eye strain and Stress because of mental pressure, so a complete 3 informational collection was gathered from each subject. For 8 subjects there were $8 * 3$ datasets to prepare the system. Yield comprise of three distinctive state. Highlights removed from the staying 4 subject, whose sign were gathered condition A were utilized to test both system. Figure 5 demonstrate the flowchart of proposed system.

Next we applied the two best settings (1-max pooling with position markers and 3-max pooling without) on all word and syllable-level undertakings. The numbers for word-based pitch emphasize recognition are equivalent and included for examination. Over all undertakings, 1-max pooling performs somewhat superior to anything 3-max pooling, to be specific around 1-2 rate focuses. The main special case is syllable-based pitch highlight recognition, where this distinction is bigger.

Syllable-level pitch highlight identification is more troublesome than word-based pitch emphasize recognition for different reasons. To begin with, the classes are less adjusted, which makes it harder to demonstrate the minority class. Second, since the words are essentially supplanted by syllables in this case, significantly less setting is given. Another explanation, accepting that the model can learn span, might be that the model can't utilize any relationships to word length that could be credited to word character or grammatical form. We additionally note that the CNN model had been streamlined for word-level applications, while the point of these tests is a confirmation of idea, which doesn't require cutting edge execution. 


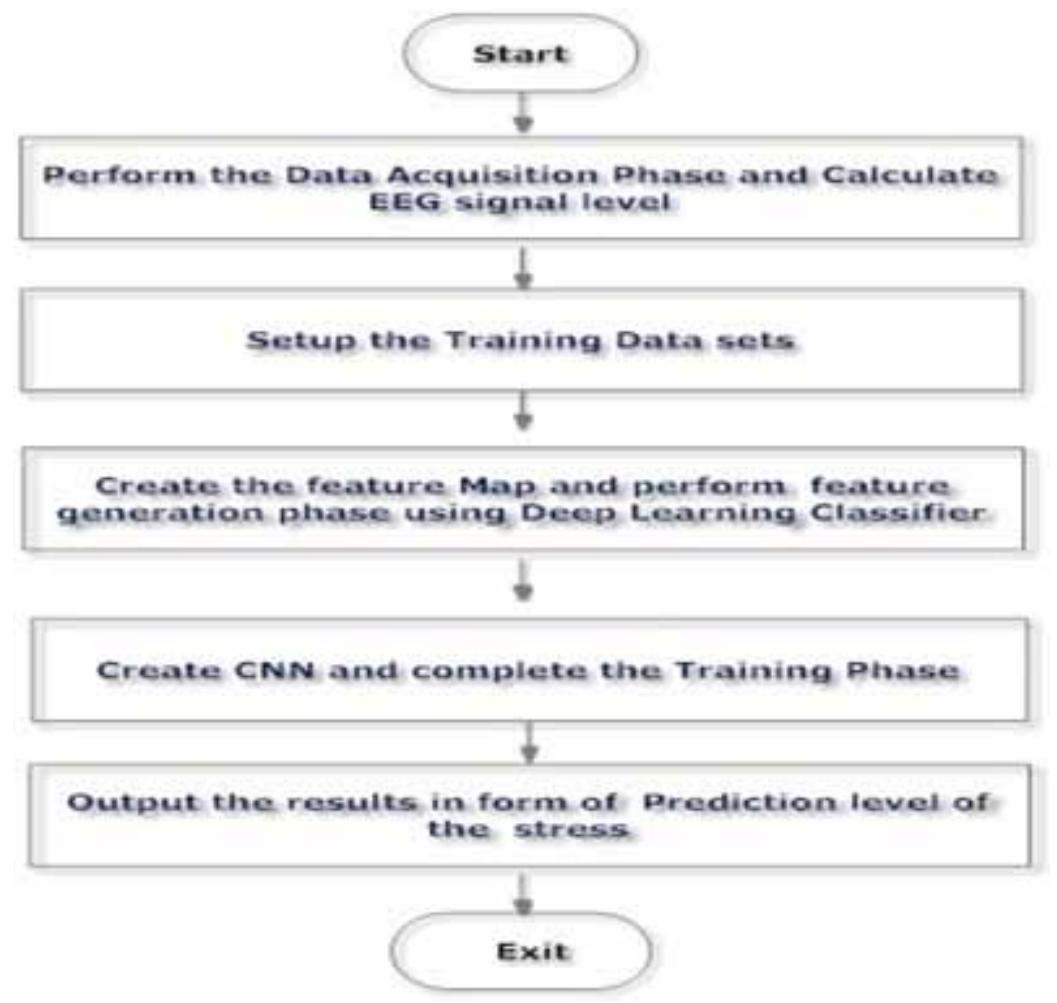

Lexical pressure identification is a simpler undertaking, which is incompletely because of the way that the classes are increasingly adjusted. Pitch emphasize recognition is encouraged when just focused on syllables are considered. For this situation, the model doesn't need to figure out how to recognize unstressed and in this manner unaccented syllables, which extraordinarily decreases the quantity of negative models in the information. For the characterization of pitch highlight types, we limit the quantity of data points further and consider just syllables which convey a positive name. The three-way characterization is significantly more troublesome than the twofold qualification not just due to the multi-class learning issue however additionally since the $\mathrm{H}^{*}$-class makes up around half of the names and in this way comprises a relatively vast lion's share class.

\section{RESULTS}

For the purpose of the performance of the proposed system for predicting the level of operational stress based of CNN, the following parameters are being judged as given below:

- $\quad$ Error rate (ER)

- False acceptance rate (FAR)

- False response rate (FRR)

It could be incited that ER, FAR, and FRR was equivalent to the distinction among one and precision, affectability and explicitness separately. Figure 6 highlighted the performance metrics of the proposed algorithm. 


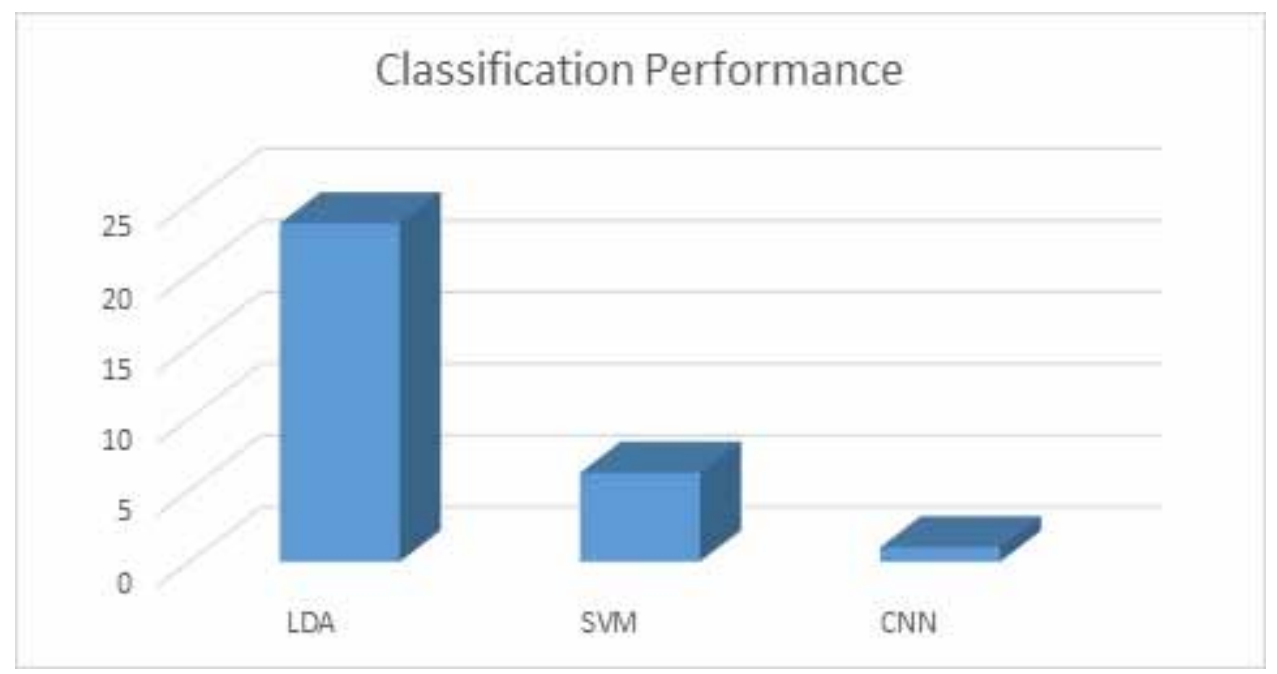

To look at the exhibition of distinguishing pressure utilizing different techniques, single direction investigation of difference (ANOVA) was utilized in this examination (Figure 7).

\subsection{Performance Comparison}

When all is said in done, CNN beat all the customary techniques (Fig. 3), among which the mix of the considerable number of highlights (Comb) accomplished the best execution. In particular, CNN accomplished the least ER at $16.5 \%$, which was $6.4 \%$ and $34.8 \%$ lower than the best and most exceedingly awful execution of the customary techniques, Comb with SVM and LH with SVM, individually.

The aftereffects of the examination for each assignment are appeared. For word-based pitch complement discovery (1), the direct model yields a modestly solid match (0.64). Contrasted with

Figure 7. Classification performance based of FAR

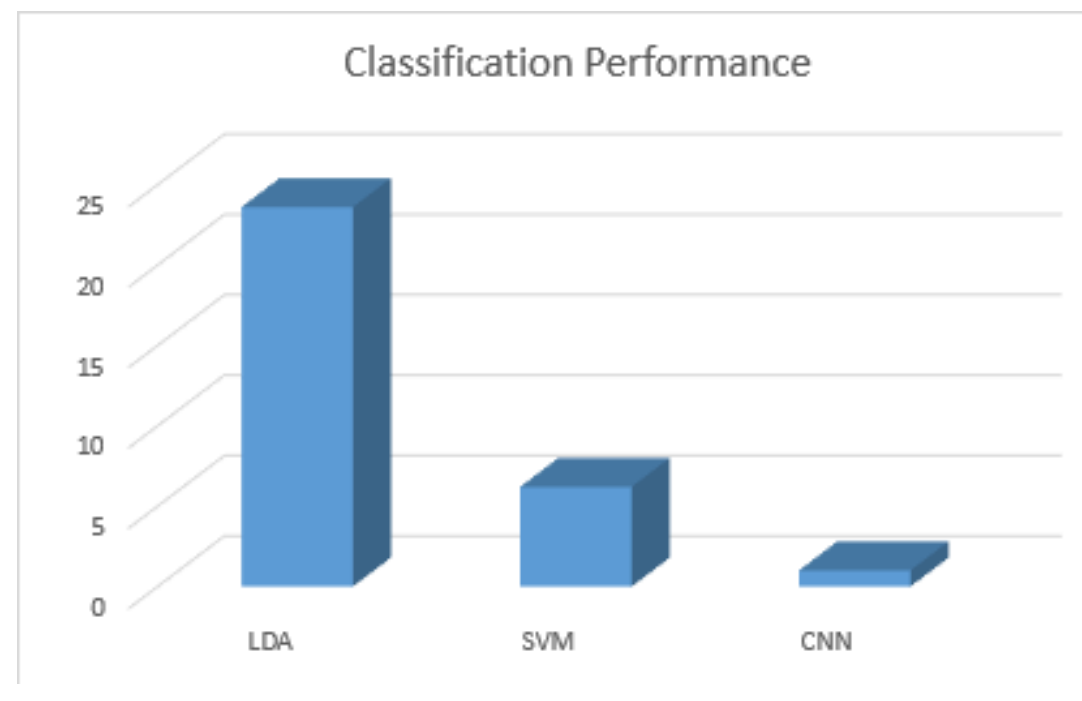


this, the R2 for the term of the present syllable is lower $(<0.50$ in errands $2-4)$, yet at the same time sufficiently high to be viewed as an estimated fit. In view of this outcome, we reason that the CNN can learn term, regardless of whether it isn't straightforwardly included as an element.

To demonstrate that this impact is because of the position markers, we included outcomes for 1-max pooling without them for this situation, is much lower. This may prompt the presumption that the position pointers just cause the CNN "to overlook" the unique situation, be that as it may, our past trials demonstrated this performed superior to when not utilizing any specific circumstance by any stretch of the imagination.

Crosswise over errands, the relationship (r) between the term of wcur and the objective name shows up to clarify how well the previous is anticipated by the straight models. It additionally demonstrates that term is more demonstrative of pitch emphasizes for words than it is for syllables, which is likely because of a lower variety in syllable length. By the by, the outcomes mirror the way that there is a distinction long among emphasized and non-complemented syllables, notwithstanding when considering just pushed ones. Strikingly, length seems, by all accounts, to be less significant for recognizing lexical pressure. There is no relationship between the syllable term and target mark for the undertakings that group given pitch emphasize types (5-6), and hence the CNN highlights don't seem to encode a lot of this data.

When utilizing 1-max pooling, the CNN cannot be said to gain proficiency with the length of the two setting words, since R2 is exceptionally low. For 3-max pooling, be that as it may, these numbers are expanded. For this situation, the CNN highlights show proof of containing span data for each of the three information words, yet comparative to 1-max pooling, the length of this factor yields the best fit. This is a fascinating outcome, since there were no position pointers utilized in this setting. In this way, the CNN seems to have naturally scholarly highlights relating for the most part to the "right" word or syllable.

In this paper, we portrayed examinations and results demonstrating that a CNN-based pitch highlight finder can learn term data all alone, despite the fact that the info comprises of basic outline put together highlights without unequivocal data with respect to word length. This likewise holds for syllable based pitch highlight and lexical pressure recognition as long as it explains the individual undertaking. We thought about two pooling techniques and demonstrated that pooling over every one of the three info words or then again syllables, instead of straightforward 1-max pooling, builds the measure of setting data caught by the CNN. Most data that is found out, be that as it may, relates to the present word or syllable. In this investigation, we concentrated uniquely on the learning of term. We are at present examining what data got from the edge based acoustic highlights is encoded in the CNN yield.

So far we have just given proof that the CNN yield contains explicit data that is the length of syllables and words. What these analyses can't respond to is the issue of how precisely this data is encoded in the last element portrayal and how it is educated. This is a momentum challenge for research on neural systems and is as of now pulling in extensive consideration. For our situation, this work comprises an initial step that adds to our comprehension of neural-arrange based models of prosody.

\section{DISCUSSION}

This investigation exhibited that $\mathrm{CNN}$ can fundamentally improve the ongoing recognition of intense psychological pressure contrasted with the traditional HRV based strategies $(\mathrm{p}<0.01)$. To explore the data $\mathrm{CNN}$ abused for pressure estimation, the initiation contrasts between the pressure state and the rest condition of the initial five layers were exhibited (Figure 5), and the grouping execution with the contribution of various recurrence groups was determined (Figure 6). The actuation contrast guide demonstrated that there was noteworthy distinction among stress and rest in the yield relating to the recurrence band over $2 \mathrm{~Hz}(\mathrm{p}<0.05)$. The exhibition correlation demonstrated that there were contrasts when the upper bound of the recurrence band was higher than $2 \mathrm{~Hz}$ or more. In any case, 
the distinctions were not huge ( $\mathrm{p}>0.05)$. This demonstrated the data in frequencies higher than 2 $\mathrm{Hz}$ may assume a job in pressure estimation of the CNN utilized in this examination, yet it was not basic. The primary data CNN caught was still principally from the recurrence band beneath $2 \mathrm{~Hz}$. Despite the fact that recurrence band over $2 \mathrm{~Hz}$ was not underscored in customary techniques, in light of the aftereffects of this investigation, its significance in stress estimation still should have been additionally investigated.

So as to enable the members to loosen up themselves in the rest session, tuning in to music was embraced for the solid impact on expanding unwinding. The brisk unwinding when the test would lessen the predisposition of the information in rest sessions, and henceforth expanded the dependability of the outcomes in this examination. The work of CNN likewise gave another approach to gain proficiency with the qualities of stress. For the customary techniques, the highlights utilized primarily relied upon the entrenched physiological hypothesis of the impact of pressure, for example, the harmony among thoughtful and parasympathetic sensory systems. Since CNN has the programmed highlight learning capacity, it empowered the analysts to investigate the attributes of worry from the data CNN caught, which may then again support the scientists, for example, researcher and physiologists to gain more bits of knowledge the physiological impact of pressure. As a matter of course, the limit of the likelihood was set to 0.5 . Through enhancing the likelihood limit in the preparation stage, the arrangement execution of stress information, subsequently the general pressure discovery execution, could further be improved.

\section{CONCLUSION AND FUTURE SCOPE}

This examination contrasted the exhibition of CNN and six ordinary HRV-based strategies in intense intellectual pressure discovery, utilizing just 10s of ECG information. Stress was instigated by mental figuring for twenty members. Not quite the same as past examinations with minutes long windows, super-short worldly windows were utilized for it was profoundly attractive in numerous down to earth applications, where the continuous pressure observing was a significant component. The outcomes demonstrated that the presentation of CNN was fundamentally superior to the traditional techniques ( $\mathrm{p}$ $<0.01$ ), and the improvement was in any event $7.2 \%$. The initiation guide of each layer demonstrated the discriminative data with respect to pressure versus rest existed in the recurrence go higher than the traditional $0.4 \mathrm{~Hz}$, and profoundly focused somewhere in the range of 0.4 and $2 \mathrm{~Hz}$. The result of this examination showed that it was conceivable to perform ongoing identification of intense intellectual worry from HRV, and CNN had potential in commonsense uses of HRV based pressure discovery. 


\section{REFERENCES}

Abdulsahib, G. M., \& Khalaf, O. I. (2018). Comparison and Evaluation of Cloud Processing Models in CloudBased Networks. International Journal of Simulation--Systems, Science \& Technology, 19(5).

Amelard, R. (2015). Feasibility of long-distance heart rate monitoring using transmittance photoplethysmographic imaging (PPGI) (Vol. 5). Sci. Rep.

American Psychological Association. (2015). Paying with our health. Stress Am.

Betti, S. (2018). Evaluation of an integrated system of wearable physiological sensors for stress monitoring in working environments by using biological markers. IEEE Transactions on Biomedical Engineering, 65(8), 1748-1758. doi:10.1109/TBME.2017.2764507 PMID:29989933

Billman, G. E., Huikuri, H. V., Sacha, J., \& Trimmel, K. (2015, February). An introduction to heart rate variability: Methodological considerations and clinical applications. Frontiers in Physiology, 6, 55.

Blaug, R., Kenyon, A., \& Lekhi, R. (2007). Stress at Work A report prepared for The Work Foundation. Project Report. http://www.theworkfoundation.com/downloadpublication/report/69_69_stress_at_work.pdf

Brennan, M., Palaniswami, M., \& Kamen, P. (2001). Do existing measures of Poincareé plot geometry reflect nonlinear features of heart rate variability? IEEE Transactions on Biomedical Engineering, 48(11), $1342-1347$.

Castaldo, R., Melillo, P., Bracale, U., Caserta, M., Triassi, M., \& Pecchia, L. (2015). Acute mental stress assessment via short term HRV analysis in healthy adults: A systematic review with meta-analysis. Biomedical Signal Processing and Control, 18, 370-377.

Castaldo, R., Montesinos, L., Melillo, P., James, C., \& Pecchia, L. (2019). Ultra-short term HRV features as surrogates of short term HRV: A case study on mental stress detection in real life. BMC Medical Informatics and Decision Making, 19(1), 12.

Deng, L., \& Yu, D. (2013). Deep Learning: Methods and Applications. Found. Trends® Signal Process, 7(3-4), 197-387.

Donida Labati, R., Muñoz, E., Piuri, V., Sassi, R., \& Scotti, F. (2018). Deep-ECG: Convolutional Neural Networks for ECG biometric recognition. Pattern Recognition Letters.

Gao, R., Hao, B., Li, H., Gao, Y., \& Zhu, T. (2013). Developing Simplified Chinese Psychological Linguistic Analysis Dictionary for Microblog. Proc. of BHI 2013.

Garcia, C. (2013). 12-Lead ECG: The Art Of Interpretation (2nd ed.). Jones \& Bartlett Learning.

Geng, W., Du, Y., Jin, W., Wei, W., Hu, Y., \& Li, J. (2016). Gesture recognition by instantaneous surface EMG images (Vol. 6). Sci. Rep.

Glorot, X., Bordes, A., \& Bengio, Y. (2011). Deep Sparse Rectifier Networks. Proc. of AI Statistics 2011, $315-323$.

Hjortskov, N., Rissén, D., Blangsted, A. K., Fallentin, N., Lundberg, U., \& Søgaard, K. (2004, June). The effect of mental stress on heart rate variability and blood pressure during computer work. European Journal of Applied Physiology, 92(1-2), 84-89.

Ireland, S. R., Warren, Y. M., \& Herringer, L. G. (1992). Anxiety and color saturation preference. Perceptual and Motor Skills, 75, 545-546.

Khalaf, O. I. (2020). Optimization of wireless sensor network coverage using the Bee Algorithm. Journal of Information Science and Engineering, 36(2), 377-386.

Khalaf, O. I., \& Abdulsahib, G. M. (2019). Frequency estimation by the method of minimum mean squared error and P-value distributed in the wireless sensor network. Journal of Information Science and Engineering, 35(5), 1099-1112.

Khalaf, O. I., Abdulsahib, G. M., Kasmaei, H. D., \& Ogudo, K. A. (2020). A new algorithm on application of blockchain technology in live stream video transmissions and telecommunications. International Journal of e-Collaboration, 16(1). 
Khalaf, O. I., \& Sabbar, B. M. (2019). An overview on wireless sensor networks and finding optimal location of nodes. Periodicals of Engineering and Natural Sciences, 7(3), 1096-1101.

Kusserow, M., Amft, O., \& Tröster, G. (2013). Modeling arousal phases in daily living using wearable sensors. IEEE Transactions on Affective Computing, 4(1), 93-105.

Lombardi, F., \& Stein, P. K. (2011). Origin of heart rate variability and turbulence: An appraisal of autonomic modulation of cardiovascular function. Frontiers in Physiology, 2.

Lu, H. (2012). StressSense: Detecting Stress in Unconstrained Acoustic Environments Using Smartphones. Proc. of UbiComp 2012.

McCraty, R., \& Zayas, M. A. (2014). Cardiac coherence, self-regulation, autonomic stability and psychosocial well-being. Frontiers in Psychology, 5(SEP).

McDuff, D., Gontarek, S., \& Picard, R. (2014). Remote measurement of cognitive stress via heart rate variability. 2014 36th Annual International Conference of the IEEE Engineering in Medicine and Biology Society, 2957-2960.

Melillo, P., Bracale, M., \& Pecchia, L. (2011). Nonlinear Heart Rate Variability features for real-life stress detection. Case study: Students under stress due to university examination (Vol. 10). Biomed. Eng. Online.

Moriguchi, A. (1992). Spectral change in heart rate variability in response to mental arithmetic before and after the beta-adrenoceptor blocker, carteolol. Clinical Autonomic Research, 2(4), 267-270.

Ngiam, J. (2011). Multimodal deep learning. Proc. of ICML 2011, 689-696.

Oskoei, M. A., \& Hu, H. (2008, August). Support vector machine-based classification scheme for myoelectric control applied to upper limb. IEEE Transactions on Biomedical Engineering, 55(8), 1956-1965.

Pan, J., \& Tompkins, W. J. (1985). A Real-Time QRS Detection Algorithm. IEEE Transactions on Biomedical Engineering, BME-32(3), 230-236.

Pecchia, L., Castaldo, R., Montesinos, L., \& Melillo, P. (2018). Are ultra-short heart rate variability features good surrogates of short-term ones? State-of-the-art review and recommendations. Healthcare Technology Letters, 5(3), 94-100.

Pelletier, C. L. (2004). The effect of music on decreasing arousal due to stress: A meta-analysis. Journal of Music Therapy, 41(3), 192-214.

Pennebaker, Booth, \& Francis. (2007). LIWC2007: Linguistic inquiry and word count. LIWC.

Rajesh, G. (2020). A statistical approach for high order epistasis interaction detection for prediction of diabetic macular edema. Informatics in Medicine Unlocked, 20, 100362.

Rajesh, G., Mercilin Raajini, X., Ashoka Rajan, R., Gokuldhev, M., \& Swetha, C. (2020). A Multi-objective Routing Optimization Using Swarm Intelligence in IoT Networks. In S. L. Peng, L. Son, G. Suseendran, \& D. Balaganesh (Eds.), Intelligent Computing and Innovation on Data Science. Lecture Notes in Networks and Systems (Vol. 118). Springer.

Rajesh, G., Ramakrishnan, T., Shreevignesh, S., Vinayagasundaram, B., \& Raajini, X. M. (2018). Achieving QoS in GSM Network by Efficient Anomaly Mitigation and Data Prediction Model. 2018 Tenth International Conference on Advanced Computing (ICoAC), 355-360. doi:10.1109/ICoAC44903.2018.8939086

Richard, S. (2006). Lazarus, "Stress and emotion: A new synthesis. Springer Publishing Company.

Ruiz, J. T., Pérez, J. D. B., \& Blázquez, J. R. B. (2019). Arrhythmia detection using convolutional neural models. Advances in Intelligent Systems and Computing, 800, 120-127.

Salahuddin, L., Cho, J., Jeong, M. G., \& Kim, D. (2007). Ultra short term analysis of heart rate variability for monitoring mental stress in mobile settings. Annual International Conference of the IEEE Engineering in Medicine and Biology - Proceedings, 4656-4659.

Saleem, S. (2012). Automatic Detection of Psychological Distress Indicators and Severity Assessment from Online Forum Posts. Proc. of COLING 2012, 2375-2388. 
Selvaraj, N., Jaryal, A., Santhosh, J., Deepak, K. K., \& Anand, S. (2008). Assessment of heart rate variability derived from finger-tip photoplethysmography as compared to electrocardiography. Journal of Medical Engineering \& Technology, 32(6), 479-484.

Shultis. (2013). Effects of music therapy vs. music medicine on physiological and psychological parameters of intensive care patients: A randomized controlled trial. Dissertation Abstracts International: Section B: The Sciences and Engineering, 73(10-B).

Smith, J. C., \& Joyce, C. A. (2004). Mozart versus new age music: Relaxation states, stress, and ABC relaxation theory. Journal of Music Therapy, 41(3), 215-224.

Srivastava, N., \& Salakhutdinov, R. (2012). Multimodal learning with deep Boltzmann machines. Proc. of NIPS 2012, 2231-2239.

Tabar, Y. R., \& Halici, U. (2017). A novel deep learning approach for classification of EEG motor imagery signals. Journal of Neural Engineering, 14(1).

Teixeira Ribeiro, R., \& Silva Cunha, J. P. (2018). A regression approach based on separability maximization for modeling a continuous-valued stress index from electrocardiogram data. Biomedical Signal Processing and Control, 46, 33-45.

Ulrich-Lai, Y. M., \& Herman, J. P. (2009). Neural regulation of endocrine and autonomic stress responses. Nature Reviews. Neuroscience, 10(6), 397-409. doi:10.1038/nrn2647 PMID:19469025

Vincent, P., Larochelle, H., Bengio, Y., \& Manzagol, P. A. (2008). Extracting and composing robust features with denoising autoencoders. Proc. of ICML 2008, 1096-1103.

Wang, X., Jia, J., Liao, H., \& Cai, L. (2012). Affective image colorization. Journal of Computer Science and Technology, 27(6), 1119-1128.

Wang, X., Jia, J., Yin, J., \& Cai, L. (2013). Interpretable aesthetic features for affective image classification. Proc. of ICIP 2013, 3230-3234.

Xiang, Y., Lin, Z., \& Meng, J. (2018). Automatic QRS complex detection using two-level convolutional neural network. Biomedical Engineering Online, 17(1).

Xue, Y. (2013). Towards a micro-blog platform for sensing and easing adolescent psychological pressures. Proc. of UbiComp 2013.

Zhai, X., Jelfs, B., Chan, R. H. M., \& Tin, C. (2017). Self-recalibrating surface EMG pattern recognition for neuroprosthesis control based on convolutional neural network. Frontiers in Neuroscience, 11(JUL).

Zhang, Q., \& Zhou, D. (2018). Deep Arm/Ear-ECG Image Learning for Highly Wearable Biometric Human Identification. Annals of Biomedical Engineering, 46(1), 122-134.

Surjeet Dalal $(P h D)$ is working as Associate Professor in SRM University, Delhi-NCR, Sonipat, Haryana. He has more than 12 years of teaching experience of teaching. His current research area is Artificial Intelligence and Cloud Computing. He has presented more than twenty papers in the national/international conferences. He has published more than thirty papers in the national and international journals. He has published 6 patents and 3 books \& submitted 2 research project in DST \& DBT.

Osamah Ibrahim Khalaf is a senior Engineering and Telecommunications Lecturer in the School of IT. He has 15 years of university-level teaching experience in computer science and network technology. 